\title{
Managing the complexity of societal needs in a nuclear emergency situation: towards further experts collaboration for the "enlightened protection" of populations
}

\author{
Y. Marignac ${ }^{1}$, J. Hazemann ${ }^{2}$ and S. Baudé \\ ${ }^{1}$ World Information Service on Energy Paris (WISE-Paris), 31-33 rue de la Colonie, 75013 Paris, France. \\ 2 EnerWebWatch, 45 allée des deux cèdres, 91210 Draveil, France. \\ 3 Mutadis, 5 rue d'Alsace, 75010 Paris, France.
}

\begin{abstract}
Expert-to-expert interactions in an emergency and post-emergency situation could be a somehow underrated, nevertheless crucial factor to meet the conditions and means for relevant and trustworthy information of the public. Improvement could therefore come through a better shared understanding by experts of their various positions, competencies, and roles - a mapping of which appears to be a useful tool for building this understanding, through improvement of trust and respect of diversity within the broad variety of experts involved, and through more intense, diversified and regular networking practices. Although they cannot be expected to form a consistent social group, experts understand they are sharing a joint responsibility, tied to their collective social function of bridging the complexity of a nuclear emergency and post-emergency situation with the complexity of societal needs arising from that situation. This should drive them to improved and reinforced collaboration under the shared objective of contributing to the "enlightened protection" of populations.
\end{abstract}

Keywords: experts / expertise / social responsibility / trust / networking

\section{Introduction}

It is widely recognized that experts have an essential role to play in the management of a nuclear emergency situation and the following recovery. However, the analysis of the efficient delivery of expertise to decision makers, to the media and to the public in such situations tends to focus on external factors to this delivery, such as a possible lack of reliable information available to experts, or the forecasted low public understanding of issues, attributed to the lack of scientific and technical culture. This vision, crossed by some controversies, most importantly leaves aside internal factors, relating to the way experts behave and interact, that could also be of crucial importance.

The experts, as a group, actually stand between the complexity of a nuclear emergency and post-emergency situation and the complexity of societal needs arising from that situation. This calls for a large number of various experts, with different competencies, experiences and social positions, to interact with society in very diverse ways. This in turn points to the need for a specific analysis of the way experts, as a group, could improve their management of this complexity, and therefore prepare to it.

In the global framework of the PREPARE project, a specific work was dedicated to emergency and post-emergency expertise networks interactions (Hazemann et al., 2016). A systemic approach was developed to address those internal factors. The analysis gradually focused on the following frame of issues:

- What are the different situations of interactions involved, how could they be characterized, and how could this help to improve the response of experts to the needs of society?

- What are the conditions of trust, and more specifically what could experts do, as individuals or collectively, to improve the trust?

- What kind of networks and interactions exist or would be needed, how can experts' practices be improved to better respond to the needs of society?

\section{Mapping as a tool}

One part of the complexity of responding to societal needs in nuclear emergency and post-emergency situations - beyond the diversity of societal needs themselves - is the diversity of the expertise resource arising from individuals enjoying various statutes and positions, and the variety of situations in which these individuals must deliver according to the needs. The range of situations of experts, from the official representatives of institutional bodies to self-trained isolated individuals, is so large that it is virtually impossible to consider them as a group, in the sense of a social one. Nevertheless, they have-or should have-two things in common. The first one is that they produce expert views, which differ from common 
opinion by their refutable construction. The second one is that they are not self-defined experts, but are entitled this role by at least some of the stakeholders involved.

This broad definition of the experts involved in a nuclear emergency and post-emergency situation goes beyond a more restrictive a priori representation of experts that often prevails. It is important for the quality of processes that both the general public and concerned stakeholders on one hand, and the various experts themselves on the second hand, develop a better understanding of the variety of the experts involved, of the reasons why they and the way they are involved. An open mapping can therefore represent a valuable tool to range the situations encountered according to two axes:

- the first one relates to the position in which an expert finds itself in the process, which depends both on its statutory position (institutional or not, member of an agency, domestic or foreign TSO, academic, consultant, etc.) and on the role that he/she has to play (understanding and characterizing, explaining, delivering information, advising, criticising, etc.), taking into account that his/her role could fit or contradict with the requirements attached to his/ her status;

- the second one deals with the variety of competencies of experts potentially involved (nuclear safety and radiological protection, but also geosciences, mechanics, agronomy, economics, social sciences, etc.), and the expand of topics arising in the course of the emergency and post-emergency situation, taking into account that the competency of an expert and the issues he/she is asked to address could also fit or contradict his/her field of competency.

This mapping should not be seen as a comprehensive exercise, but rather a tool to provide an open framing of the system through which expertise is delivered and the mechanisms involved, illustrating the complexity of interactions in the context of the Fukushima crisis or another nuclear accident, and the different issues at stake.

It is in that sense a way to develop a shared understanding of this complexity amongst experts and stakeholders and raise their awareness of the difficulties encountered. This shared understanding increasingly emerges as a key to better preparedness.

\section{Trust and interaction}

The research also emphasized the importance that relations between experts can have regarding their collective contribution to fulfilling the needs which society expects them to fulfil. A part of it involves the development of better networking and interacting practices. But trustworthiness of the diversified expertise that they collectively provide firstly depends on the reflection in society of the trust experts manage to build among themselves. There is a need to recognize that interactions amongst experts are not only about contents, but also involve personal values, and therefore personal respect or contempt, which implies that better experts networking is not only about smoother practices, but also about building mutual recognition between experts of various position and origin (EnerWebWatch, 2013).
Beside pre-established official and institutional channels, experts relied on their networks to get or provide information: individual exchanges, diffusions lists, closed discussion lists, and meetings. In some cases, this implied structured in "real time" information monitoring / collaborative analysis of the events as they were evolving, coupling systematic information monitoring (documents, institutional sites in different countries, webcams, social media, etc.) and selection, with participation of experts on hold for reactions at the international level.

Those networks allowed for complementarity of approaches, competences, expertise (from very specific to system analysis), and for flexibility.

Other experts dealing with specific issues arising as the situation evolved (problems linked to the use of salted water to cool down the reactors for instance) were identified and their expertise could be added to those of other participants (directly or through contacts).

If the documentation management side needs to be appropriately addressed, the real added value of those networks remains the fact that they enabled experts to produce collective questioning, analysis, assessments and expertise. They proved very efficient to convey information and expertise amongst their members (in a broad sense), and also contributed to reliable assessments of the evolving situation and issues at sakes.

Our research mostly focussed on interactions between institutional and non-institutional experts/networks, and amongst the later.

A clear, and not so surprising result, was that "interactions" between different networks, or type of networks, were obviously made easier, if at all, through previous connections, working experiences, and even more through individuals already involved in different types of networks. Personal relations helped identify relevant interlocutors and contact them, without going through the hierarchical chain.

But in the context of institutional/non-institutional networks, interactions were often understood as mere information sharing. Access to or circulation of information took place through individual contacts (in particular between different channels), for institutional/non institutional exchanges, as well as within institutions themselves, i.e. also within formalised environments.

They usually missed the point of a collective process to face the crisis. Although some exchanges worked well, experts expressed the lack or inappropriate levels of interactions, missed opportunities, and a general feeling of unbalanced relation:

- A lack of productive confrontations and sound exchanges on the content.

- A profound disappointment on production of "unused" expertise.

- Difficulty to acknowledge the value of the different agendas and the importance of the role of the different actors, the value and usefulness of input from other experts, and in particular from "critical" experts.

This confirms the need to address those questions in a more systemic way. If personal relations are important, issues at stake call for a more thorough reflection on the role of experts and their interactions. 
In that respect, there is a need to make a clear distinction between:

- addressing the interaction channels (personal, organised, between networks, etc.) and proposed mechanisms to improve those interactions, and

- addressing the nature and the objectives of interactions.

They should not be seen as burdens and time consuming activities, but as a common way to reinforce the efficiency of contributions of experts on an individual level and strengthen their collective capacity to fulfil the demands of society.

\section{Conclusions}

The experts, in their broad diversity, share the responsibility to deal with the complexity of a nuclear emergency and post-emergency situation in a way that fits the complexity of societal needs

They should and can share, through better understanding, a common goal of enlightened protection of populations. A "mapping" of the situations of interaction of the experts with society could help both to develop a better (and better shared) understanding of the variety and relevance of these interactions, and to improve them. It is also important for this process that personal values involved in experts judgments, and personal respect or contempt involved in expert-to-expert interactions are better acknowledged, so as to develop trustworthiness of the diverse expertise they collectively provide.

Therefore better experts networking, which goes with broader interactions and smoother practices, is not only a way to make the preparation and delivery of this collective and diverse expertise more efficient. It is also an essential mean for building mutual recognition and exchanges between experts of various position and origin, and clarifying the goals pursued.

\section{References}

EnerWebWatch (2013) PREPARE Workshop: Managing Complexity in Nuclear Accidental Situations - Experts Interacting with Experts and Society. A Collection of Plenary Presentations, Lisbon, November 2013, www.enerwebwatch.eu/Prepare-Lisbonworkshop-p22.html.

Hazemann J. et al. (2016) Emergency and post-emergency expertise networks interactions, PREPARE Deliverable D.6.10. 1.

Cite this article as: Y. Marignac, J. Hazemann, S. Baudé. Managing the complexity of societal needs in a nuclear emergency situation: towards further experts collaboration for the "enlightened protection" of populations. Radioprotection 51(HS2), S159-S161 (2016). 